\title{
Fault Simulations on a Distribution Network
}

\section{through a GUI}

\author{
Varun Jadhav, Noah Dias ${ }^{2}$, Arulselvan G' ${ }^{3}$, Ganesh Pushpakumar ${ }^{4}$ \\ Electrical and Electronics Department, Goa College of Engineering, Farmagudi, Ponda Goa, India ${ }^{1,2}$ \\ Research \& Development Department, Siemens Ltd., Verna Industrial Estate, Verna, Goa, India ${ }^{3,4}$
}

\begin{abstract}
Fault Simulations are very important to study the nature of the systems in place and thus test the Protective Relays under those conditions. Omicron modules have very high accuracy in its class of Power System simulators, not only in the steady regions but the transient states as well. This paper focuses on the Fault Simulation on a Distribution Network through MATLAB and the Omicron module. The faults are simulated on a distribution model (using the Sim Power Systems ${ }^{\circledR}$ Tool) in MATLAB. MATLAB is interfaced with the Omicron module (using CM Engine ${ }^{\circledR}$ ) for the real time testing of the relays. The GUI gives added functionality and ease of use to the user. A report generator is added, which generates a report of the simulation along with the waveforms.
\end{abstract}

Keywords: Fault Simulations, GUI, Omicron Power System Simulator, Protective Relays.

\section{INTRODUCTION}

A network made up of electrical component meant to supply, transmit and utilize the electric power is an electric power system. An example of an electric power system is the grid which is divided into the generating system, the transmission system and the distribution system. In such network systems fault localization and isolation becomes crucial for ensuring continuous supply of electricity.

Hence the need of high accuracy monitoring and sensing systems has increased. Also, the testing of such protective equipment under the normal working conditions as well as harsh conditions is of a primary importance, which is achieved through Simulations on MATLAB and injection of that data into the protective equipment using a power system simulator like Omicron CMC 356 [8][9].

The paper is organized in the following manner in Section I Introduction, Section II Electrical Faults in a Power System, Section III Protective Relays, Section IV Implementation, Section V Simulation Results, and Section VI gives a brief discussion on the paper.

\section{ELECTRICAL FAULTS IN A POWER SYSTEM}

Electric power systems have a complex \& dynamic behavior. The various types of faults interrupt its healthy operation. Examples of the chief electrical faults are phase faults, which include the balanced three phase faults, Single and Double line to ground faults.

Additional electrical faults aren't of any significance but are still to be considered. These are: a) open circuit faults occurring due to the separation of the overhead line or failure of the circuit breaker operation. b) Inter-turn faults occurring due to the breakdown of insulation or overvoltage.

Electrical faults are caused due to the overloads which are caused when the current passing through the conductor is above the permissible limit.

The faults due to real power deficit are caused as the generated power and consumed power aren't in tandem which further results in the frequency deviation, resulting in the collapsing of the grid [4][5].

The phase faults are characterized as:

1. Three Phase Fault (3L)

2. Single Line to Ground Fault (L-G)

3. Line to Line Fault (L-L)

4. Double Line to Ground Fault (L-L-G) 


\section{PROTECTIVE RELAYS}

One of the most important components of a power protection system is the relay which is a device that trips the circuit breakers when the input voltage and current signals correspond to the fault conditions designed for the relay operation. Relays in general can be classified into the following categories [6]:

1. Directional Relays: They respond to the difference in phase angle between two inputs to the relay.

2. Differential Relays: They respond to the magnitude of the algebraic sum of its multiple inputs.

3. Magnitude Relays: They respond to the magnitude of the input quantity.

4. Pilot Relays: They respond to the input signals that are sent to the relay from a remote location.

5. Distance Relays: They respond to the ratio of two input phasor signals.

Over the years the relay technology has developed and the classification based on generations is as follows [7]:

1. Electromechanical Relays: They are the first generation of relays. They use the principle of electromechanical conversion. They are rugged \& immune to electromagnetic interference. But with recent advancements, they have been turned obsolete in most areas.

2. Solid State Relay: They make use of transistors, op-amps, etc. They are more flexible with a self-check facility, consuming less power and having improved dynamic performance than the electromechanical relays. They also were smaller in size requiring less panel space.

3. Numerical Relays: Operation involves Analog to Digital conversion of currents and voltages, which are acquired from the CTs and VT which is fed to the DSP or microprocessor. These signals are then processed using the protection algorithms and the necessary decisions are taken. The advantages of a Numerical Relay are:

- High flexibility.

- Multiple functionalities.

- Self-checking and communication facility.

- Can be adaptive.

\section{IMPLEMENTATION}

The implementation deals with the model made in MATLAB using the Sim Power Systems ${ }^{\circledR}$ Tool, the GUI and the backend script of the GUI with Omicron controls [1][2][3].

A. The Model

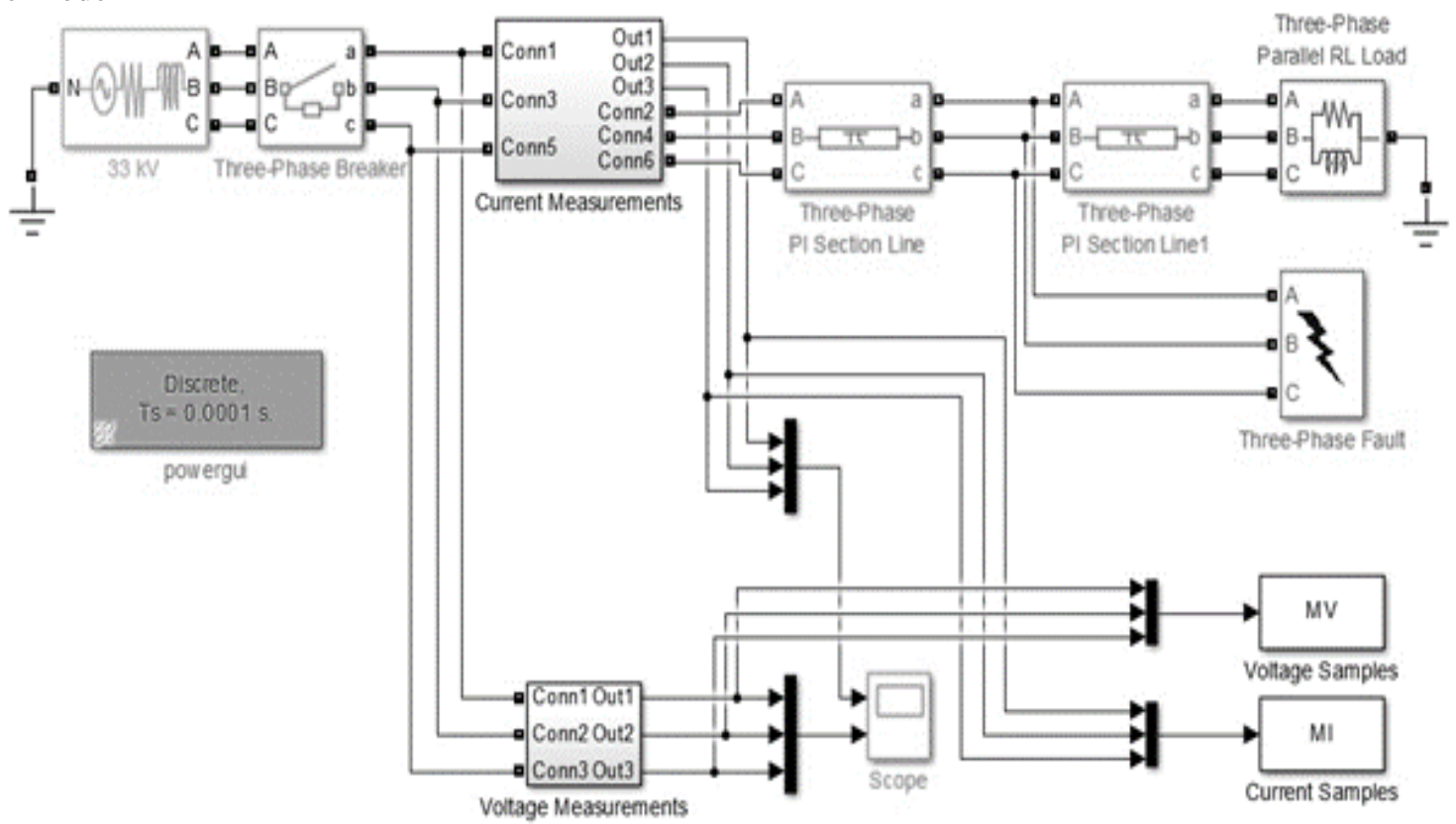

Fig 1: The Distribution Model

B. The GUI

1) Algorithm:

1. Start

2. Run the model file (.mdl/.slx)

3. Define CT \& PT Ratio 
NMAM Institute of Technology, Nitte

Vol. 4, Special Issue 2, April 2016

4. Define no. of samples, phases, sampling time(frequency)

5. Define fault \& circuit breaker operating times

6. Define system voltage, \& line lengths

7. Create COM server for Omicron \& invoke tests on it

8. Check \& confirm Omicron module association

9. Allocate memory for Current \& Voltage data from the three phases

10. Check Max \& Min values of Current \& Voltage in each phase \& if abs $(\min )>\max , \operatorname{abs}(\min )=\max$

11. Normalize max Current \& Voltage value to $32767 \&$ change matrix to array

12. Separate phase wise Current \& Voltage data into different commands

13. Initialize buffer to calculate the trip time

14. Create user defined Current \& Voltage waveform

15. Run Test \& get the trip status \& trip time of the relay

16. Delete Test \& unlock Omicron

17. Save the generated plots

18. Generate report by creating another COM server for Excel \& passing data from MATLAB to Excel

19. Convert the file into a pdf \& save the report

20. End
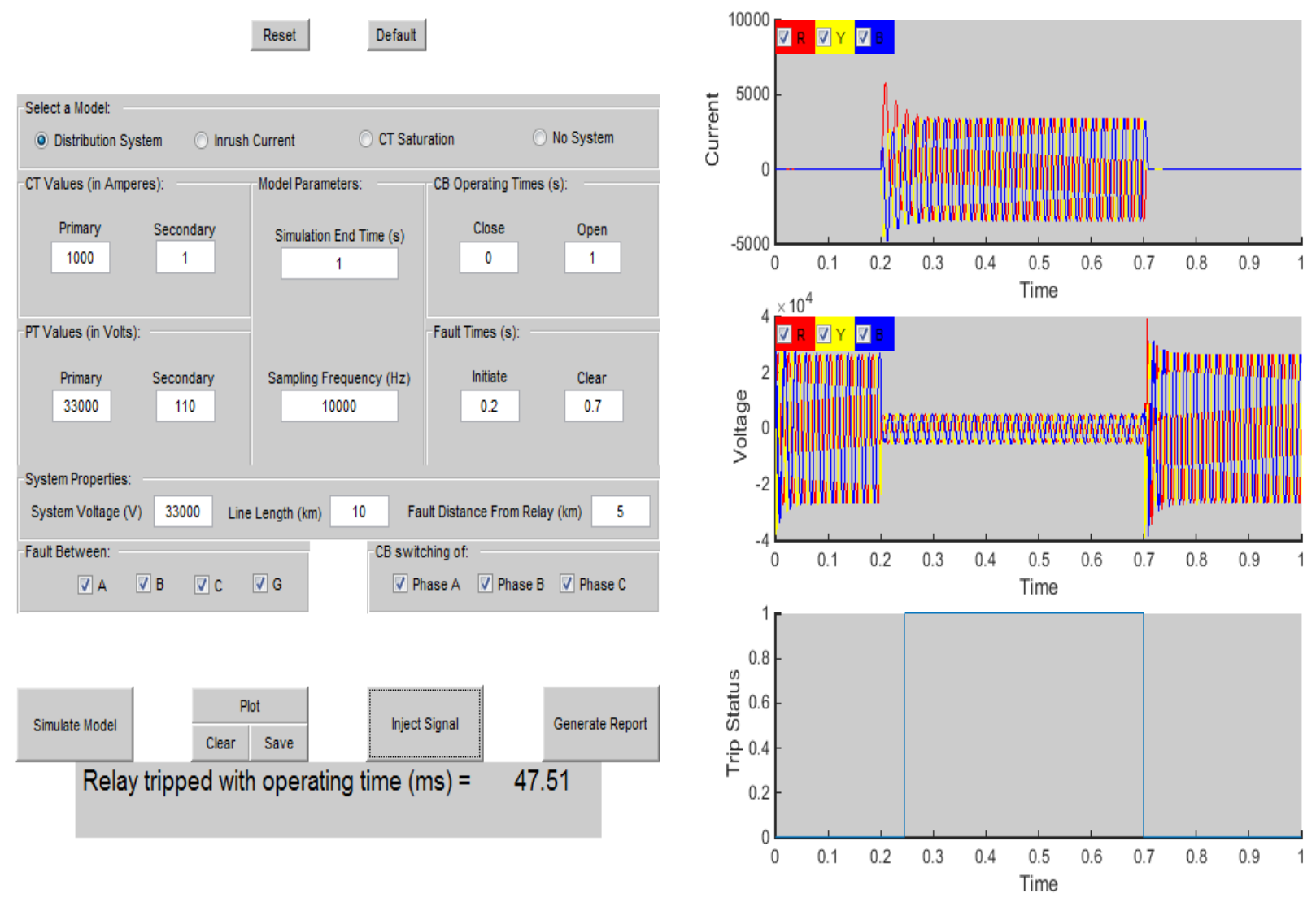

Fig 2: The GUI after simulation

2) Features of the GUI:

1. High range selectivity, thus enabling many test cases. Also, the waveforms for each phase are selectable for viewing single or multiple waveforms at a time.

2. The time axes are linked to each other allowing zoom and pan options for a better view \& comparative analysis.

3. It prompts the errors, if any, to the status bar and guides the user to the possible corrections.

4. Default values of each model are programmed which enables a user to directly simulate using default data. Also, a reset button is provided if a fresh test case is desired, thus avoiding the cumbersome, closing and reopening process.

5. The length of the line and setting of the distance of the fault can be defined and thus can be used to analyze the reach of the relay, by setting increasing distances and cross checking with trip times. 
6. The "Run" part of the GUI has been split into, "Simulate Model", "Plot", "Inject Signals" and "Generate Report" to improve functionality, allowing the user to use the GUI just to view plots, or not generate the report when not needed. Also, it is not linked to the model in a controlling fashion; which means that the model can be run separately in a standalone way, without opening the GUI.

7. From the third plot we can analyze trip status, trip time, the time of the relay contacts staying high and the effectiveness of the relay.

\section{SIMULATION RESULTS}

A. Test Cases:

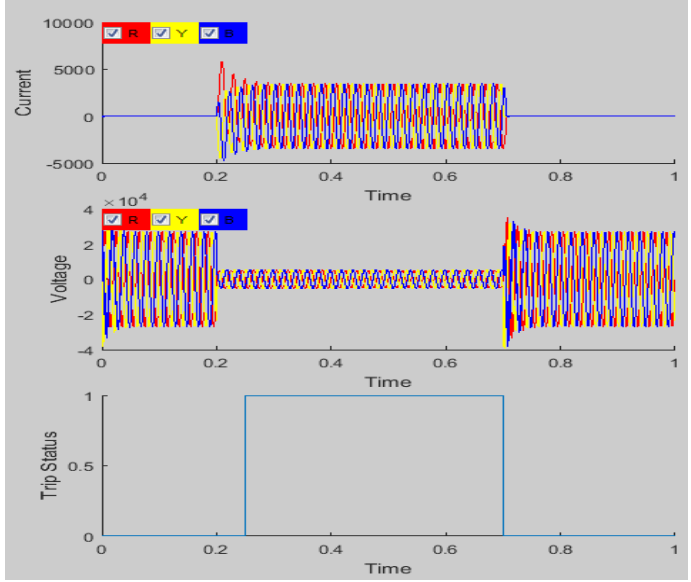

Fig 3: R-Y-B

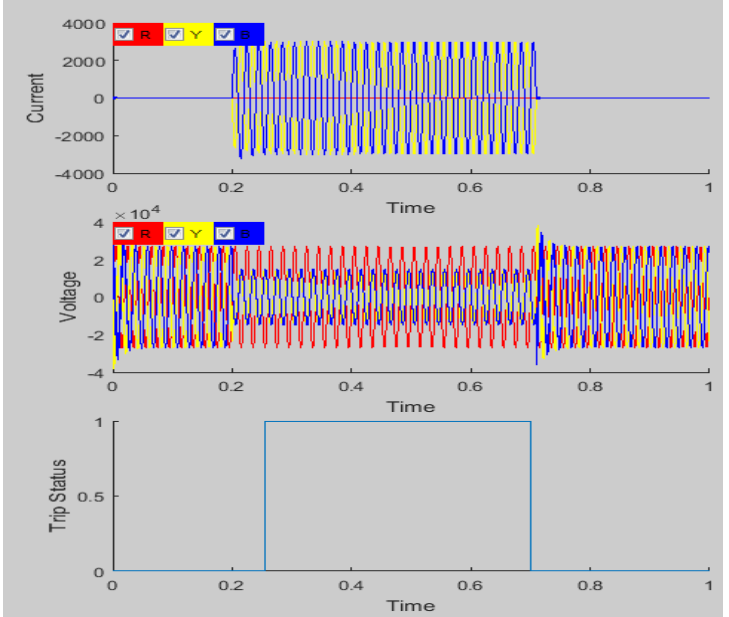

Fig 5: Y-B

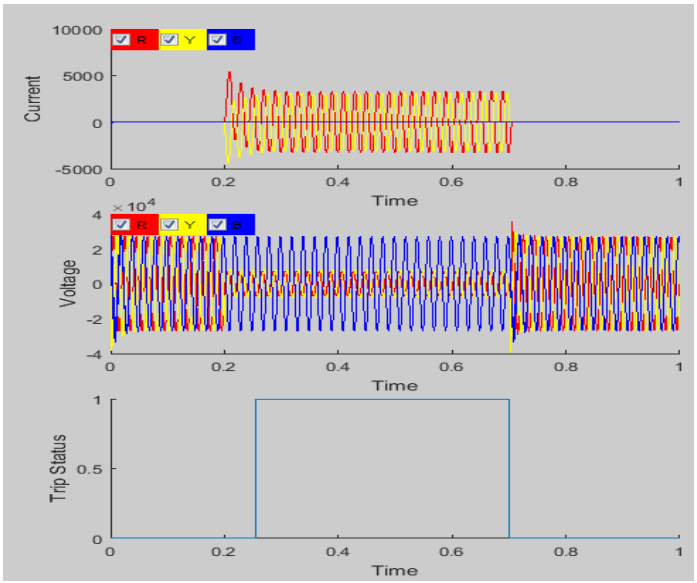

Fig 7: R-Y-G

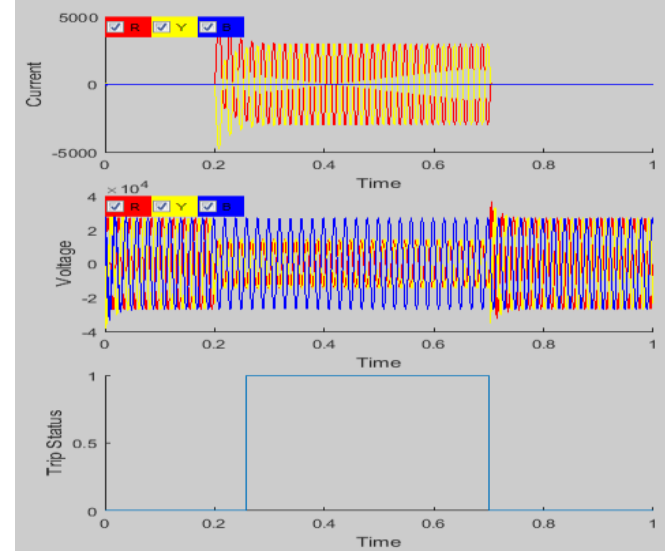

Fig 4: R-Y

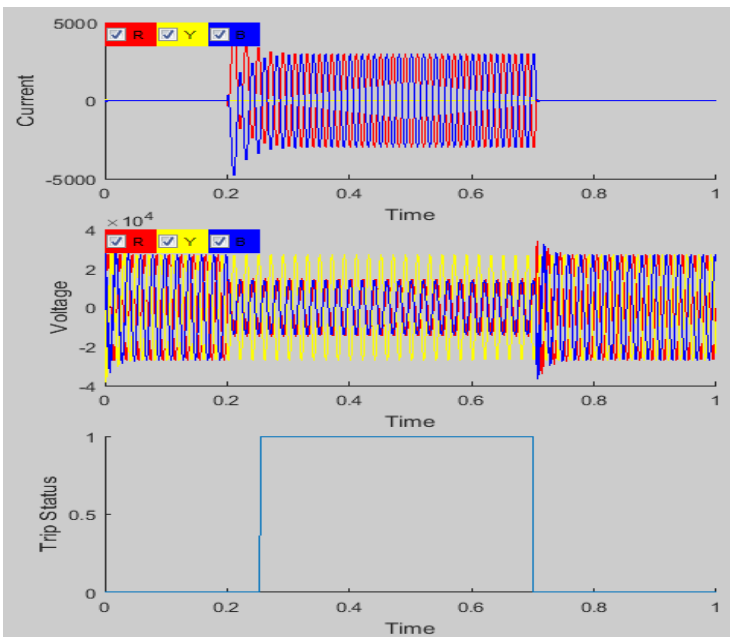

Fig 6: R-B
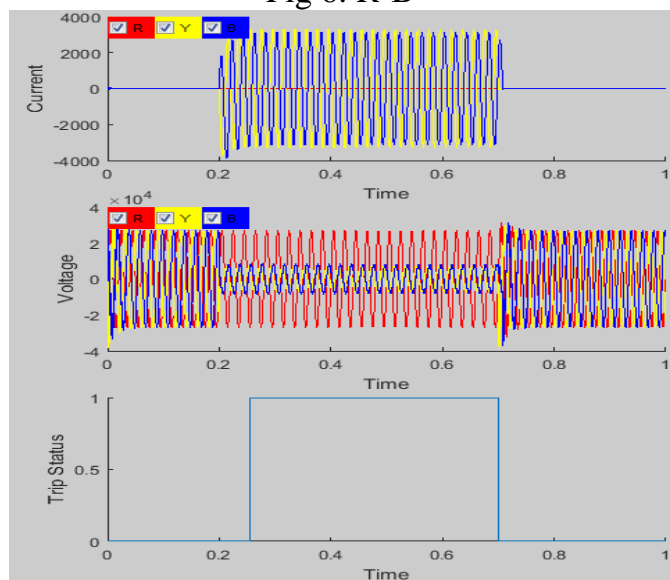

Fig 8: Y-B-G 
NMAM Institute of Technology, Nitte

Vol. 4, Special Issue 2, April 2016

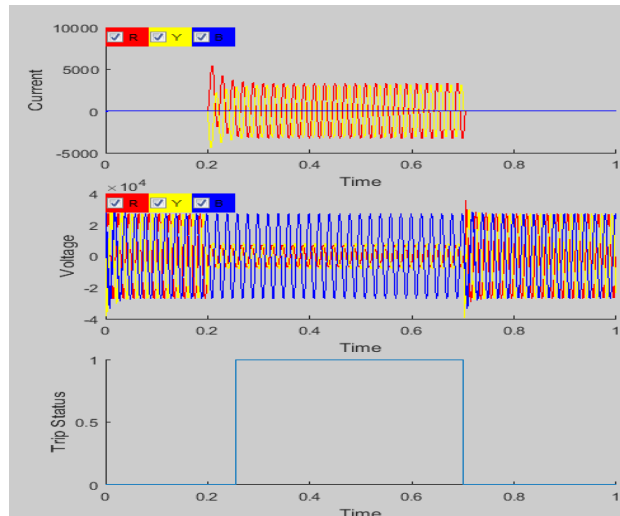

Fig 7: R-Y-G
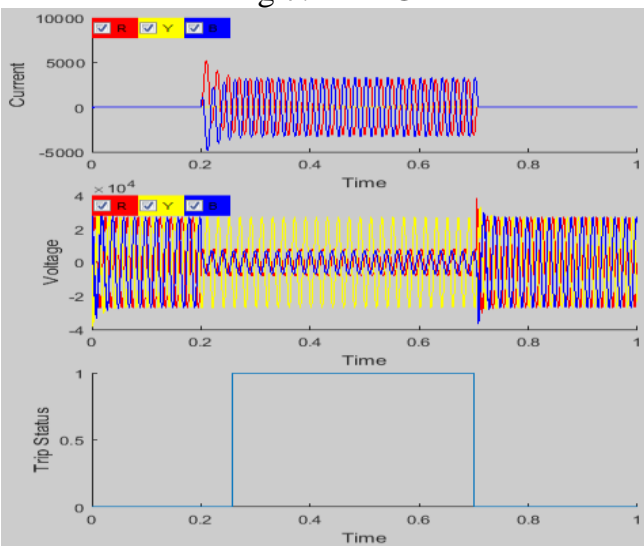

Fig 9: R-B-G

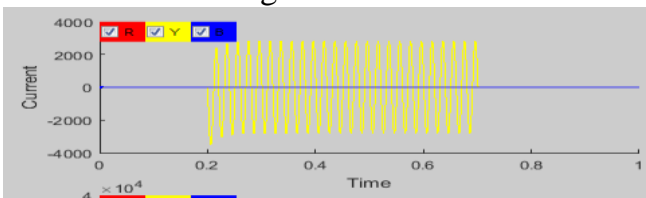

$\sqrt[4]{2^{2}}$

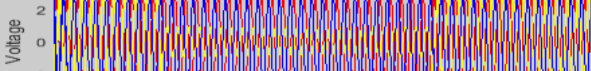

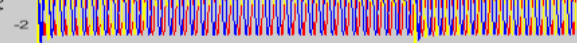

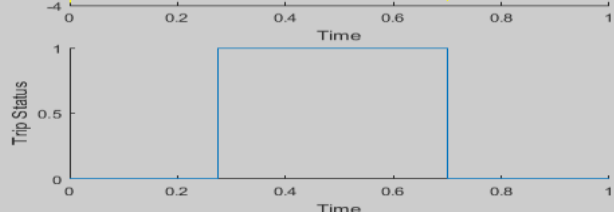

Fig 11: Y-G

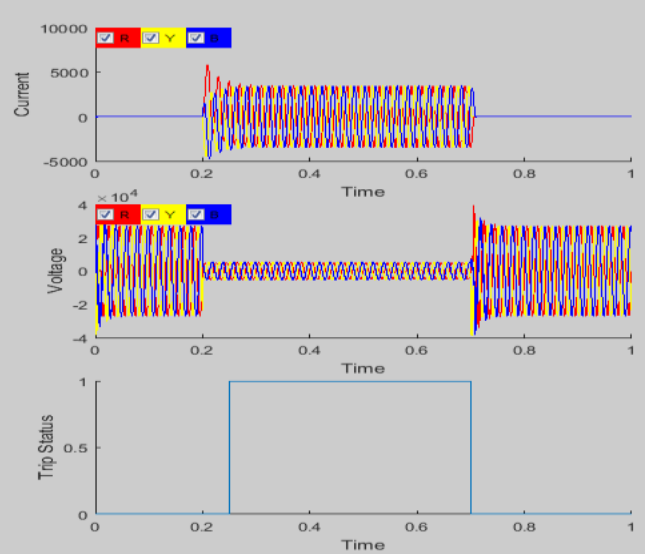

Fig 13: R-Y-B-G

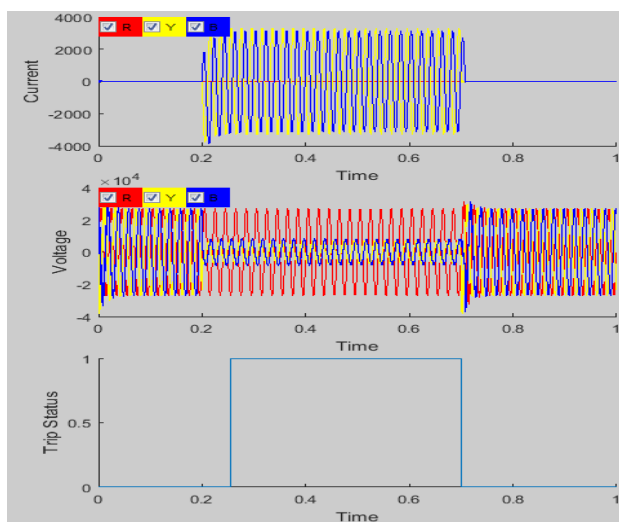

Fig 8: Y-B-G

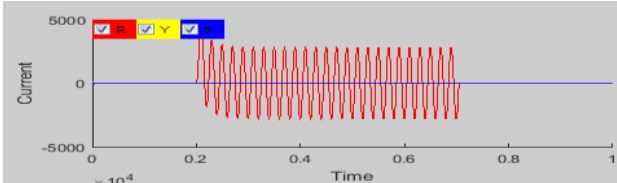

${ }_{2}^{4} \stackrel{10}{10}^{2}$
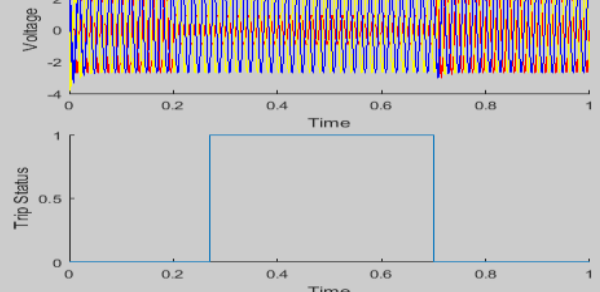

Fig 10: R-G

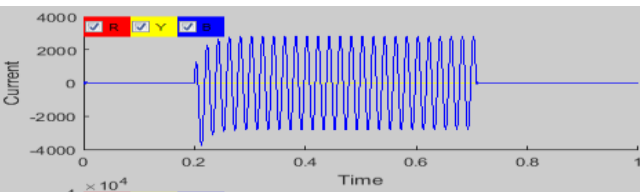

${ }^{4} \frac{x^{10^{4}}}{\sigma^{2}} \sqrt{r^{2}}$

罯
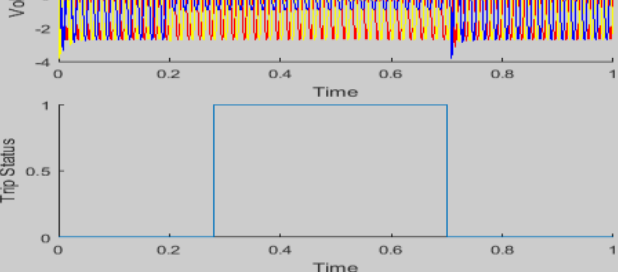

Fig 12: B-G
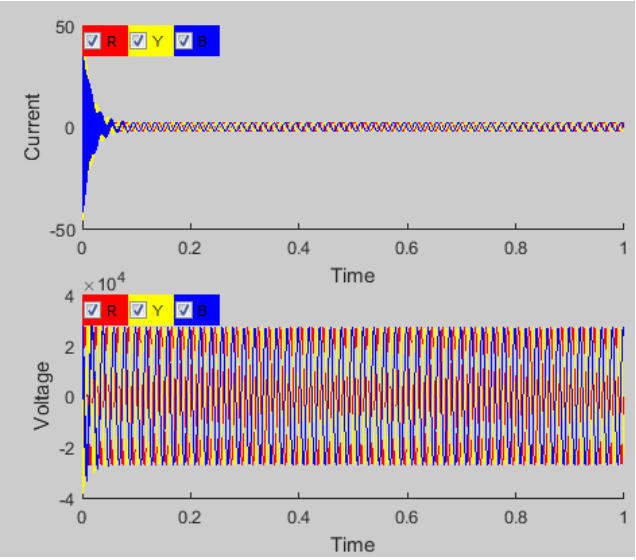

Fig 14: No Fault 
NMAM Institute of Technology, Nitte

Vol. 4, Special Issue 2, April 2016

\section{DISCUSSION}

1. Fig. 2 shows the view of the GUI upon running it. In this particular case, the Distribution system model is run for a Three Phase to Ground Fault. The simulation is done for $1 \mathrm{sec}$, so that the waveforms can be seen more clearly. The sampling frequency is taken to be $10 \mathrm{kHz}$. The system voltage is taken as $33 \mathrm{kV}$ and the line length is taken as $10 \mathrm{kms}$, with fault occurring at $5 \mathrm{kms}$. Fault is initiated at 0.2 secs and cleared at 0.7 secs. These parameters have been kept constant for other test cases as well.

2. On the right side of Fig. 2, we have the current and voltage waveforms for the given specifications. Upon injecting these signals to the relay via the Omicron module we see that the relay trips after $47.51 \mathrm{~ms}$ and the status of its coil is shown in the third graph. As this is a self-reset relay, the trip status comes back to 0 upon clearing the fault, but for a manual reset relay, it stays 1 till the reset button is pressed manually.

3. From the test cases we see that the GUI is able to simulate all kinds of faults on the line and test the relay accurately giving the trip status and the trip time.

4. The model can be ran standalone or on the GUI to view the plots. Thus making the GUI exclusive whenever needed, and showing the adaptation of the GUI to the other changes in the model as well.

\section{REFERENCES}

[1]. Omicron CMC 356 User Manual

[2]. Omicron CM Engine ${ }^{\circledR}$ User Manual

[3]. MATLAB Documentation, www.mathworks.com

[4]. Md Asaduzzaman Nur, Mehdi Hasan Sourov, M S Muhit, Md KhaledvHossain, "Fault analysis and Electrical Protection of Distribution System", Global Journal of Researches in Engineering, Volume 14, Issue 3, Version 1.0, 2014

[5]. M. Kezunovic, G. Latisko, M. Knezev, T. Popovic, "Automation of Fault Analysis: Implementation Approaches and Related Benefits," International Conference on Electrical Engineering, ICEE 07, Hong Kong, July, 2007.

[6]. Suhaas Bhargava Ayyagari, “Artificial Neural Network Based Fault Location For Transmission Lines” University of Kentucky, Master's thesis, online

[7]. NPTEL lectures, "Power System Protection", S.A. Soman, IIT Bombay

[8]. https://en.wikipedia.org/wiki/Power-system_protection

[9]. https://en.wikipedia.org/wiki/Electric_power_system 